\title{
Biodegradable Luminescent Silicon Quantum Dots for Two Photon Imaging Applications
}

\author{
Chen-An Tien ${ }^{a}, F_{\text {Folarin Erogbogbo }}{ }^{a b c}$, ,Ching-Wen Chang ${ }^{a}$, Priscilla Adjei-Baffour $^{c}$,Wing-Cheung \\ Law $^{c}$, Mark T. Swihart ${ }^{a, b} *$
}

Department of Chemical and Biological Engineering ${ }^{\mathrm{a}}$, and Institute for Lasers, Photonics and

Biophotonics $^{\mathrm{b}}$, The University at Buffalo, State University of New York

Buffalo, New York 14260-4200

Email: $\underline{\text { swihart@buffalo.edu }}$ 
Abstract

Cadmium- and lead-based quantum dots are normally coated for biological applications, because their degradation may result in the release of toxic heavy metal ions. Here, we synthesize silicon quantum dots that are expected to biodegrade to non-toxic products. A chitosan coating is used to render the silicon quantum dots stable in storage conditions and biodegradable at physiological conditions. The applications of these particles are demonstrated in cellular imaging with single and two-photon excitation. These results open the door for a new generation of silicon quantum dots that may have a wide variety of applications derived from the flexibility of chitosan.

KEYWORDS silicon, nanocrystals, quantum dots, chitosan, biodegradable, imaging 


\section{Introduction}

The discovery and investigation of light-emitting semiconductor nanocrystals (quantum dots) has led to significant demonstrations of their potential to advance biological research. ${ }^{1-2}$ However, achieving efficient imaging in a target cell population or tissue without causing any quantum dot-associated toxicity is critical for expanding their role as imaging agents. Important efforts have already been focused on reducing the toxicity of heavy metal-based quantum dots for biological applications. ${ }^{3-4}$ Among them, coating cadmium-based nanoparticles with a $\mathrm{ZnS}$ shell has been shown to reduce the toxicity of particles that enter systemic circulation. Several years ago, it was widely accepted that heavymetal-based quantum dots showed promise for future clinical applications. ${ }^{5-6}$ At the time, the focus was on quantum dots' bright emission, multiplex imaging capabilities, broad excitation spectra, photostability, and lifetime. However, the inclusion of toxic elements in these QDs has slowed their translation toward the clinic. While several studies have used of Cd-based QDs in animals with no apparent toxicity ${ }^{4}$, use of heavy metals remains undesirable in the rational design of nanoparticle imaging agents because: (1) the toxic reputation of bulk and elemental cadmium creates resistance to Cd-based quantum dots being accepted as non-toxic ${ }^{3}(2)$ extensive studies will be needed to evaluate multiple potential routes of Cd-based QD toxicity to alleviate concerns, ${ }^{4}$ and (3) although biodegradation is desirable for most imaging agents, approaches to address toxicity of Cd-based QDs generally aim to avoid biodegradation because of the resulting release of $\mathrm{Cd}$ ions. ${ }^{7}$ The need for inherently non-toxic components for quantum dots has partially motivated the development of silicon quantum dots (SiQDs). ${ }^{8-12}$ However, despite recent progress in development of SiQDs for bioimaging, further advances are required, including development of optimal encapsulation strategies, increasing its quantum yield, and creating nanoconstructs that exploit its biodegradability. Additionally, physiological factors affecting the performance and degradation of silicon nanoparticles are not yet well understood. Nevertheless, there are important biological principles that can guide the design of a desirable silicon quantum dots nanostructure. With these principles in mind, advancements have been made using SiQDs for biological applications. Sailor's group recently developed biodegradable porous silicon 
nanostructures and demonstrated tumor imaging with them. ${ }^{13}$ Although free standing silicon is expected to degrade by the same routes as porous silicon, its surface chemistry and structure are different enough for it to be considered a different nanomaterial. Kauzlarich's group has developed Mn-doped SiQDs for magnetic resonance imaging, demonstrated in macrophages. ${ }^{11}$ Tilley's group has demonstrated that silicon quantum dots are 10 times less toxic than Cd-based quantum dots and used them for cellular imaging ${ }^{14}$. We previously have designed new types of SiQD-based nanoconstructs that improved the quality, and options available for silicon based imaging agents. ${ }^{9}$ The systems are PEGylated phospholipid micelle encapsulated silicon quantum dots (MSiQDs) that are useful for cancer applications such as tumor targeting, sentinel lymphnode mapping and multiplex imaging. ${ }^{15}$ The MSiQDs were designed to make the SiQDs more stable in vivo. In them, the PEG brush hinders protein/enzyme adsorption while the SiQDs remain in the hydrophobic interior of the micelles. That approach provides very stable particles whose in vivo luminescence remains visible even after one month. However, for many applications, biodegradation after a shorter time would be preferable.

Chitosan, a naturally biodegradable, biocompatible polymer has been extensively used in biological investigations, ${ }^{16}$ including nucleic acid transfection, ${ }^{17-18}$ protein delivery, ${ }^{19}$ drug delivery, ${ }^{20}$ and quantum dot encapsulation. ${ }^{21-22}$ These prior studies of chitosan-coated quantum dots showed promise for biological imaging, however they used cadmium based quantum dots. Here, we report a biodegradable chitosan-silicon nanocomplex (CSiQDs) for imaging applications. We chose 75-85\% deacetylated low molecular weight chitosan to make a reasonably sized nanoparticles and provide adequate stabilization with a positive charge. Undecylenic acid was used to functionalize the SiQDs because of its biocompatibility, it carboxyl termination and the photoluminescence stabilization it provides for SiQDs when compared to shorter chain carboxylic acids ${ }^{23-24}$. Figure 1 schematically depicts the hydrosilylation reaction that creates the Si-C linkage and the subsequent encapsulation in chitosan. 


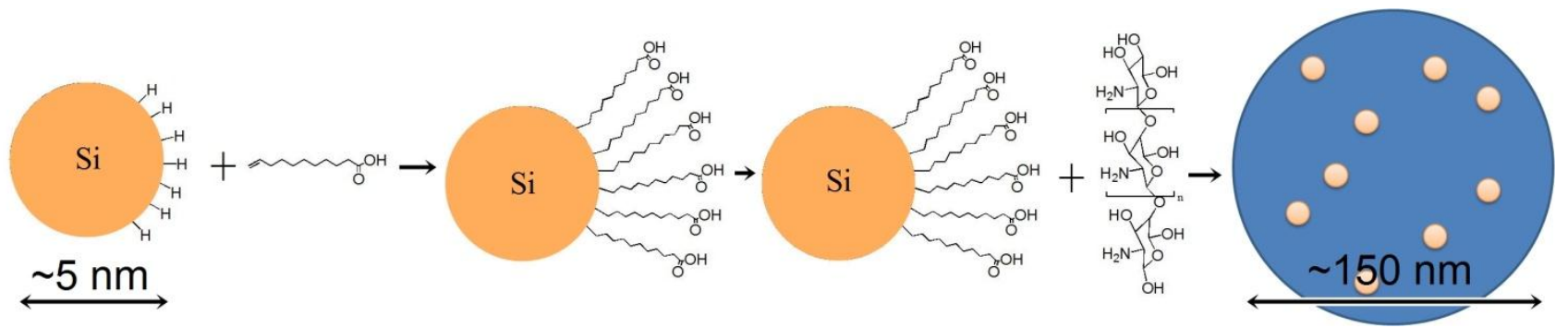

Figure 1. Schematic illustration of hydrosilylation reaction of undecylenic acid with hydrogenterminated SiQDs and the encapsulation of SiQDs with chitosan. SiQDs are represented by orange circles and chitosan is represented by the blue circle. 


\section{Results and Discussion}

Figure 1 schematically illustrates the functionalization of SiQDs with undecylenic acid and the chitosan encapsulation process. The double bond on undecylenic acid reacts with hydrogen terminated SiQDs to generate carboxyl terminated SiQDs. The amine group on each repeat unit of chitosan can coordinate with the caboxyl groups by electrostatic interaction of the partial positive charge of the amine groups and the partial negative charge of the caboxyl groups to form Si QD-embedded chitosan nanoparticles. Multiple silicon nanoparticles are trapped within each nanocomplex.

Figure 2 presents the optical properties of Si-und and CSiQDs. The PL emission peak shifted slightly from $643 \mathrm{~nm}$ to $632 \mathrm{~nm}$ after the encapsulation. Surface coatings are known to affect the emission wavelength of light emitting nanoparticles. In particular, when silicon quantum dots are in water, the shift may be attributed to a slight oxidation. The FWHM remained approximately the same. FTIR was used to confirm the attachement of undecylenic acid to the particles indicating the presence of carboxyl groups on the surface of the silicon quantum $\operatorname{dots}^{24}$. The electrostatic binding interaction was confirmed by zeta potential. The surface charge of the particles coated with chitosan is $45 \mathrm{eV}$ when meausred in water. This positive charge is attributed to the amine groups present in chitosan. Zeta potential measuments before coating the silicon with chitosan are not made because the particles are not soluble in water with the undecylenic acid coating.

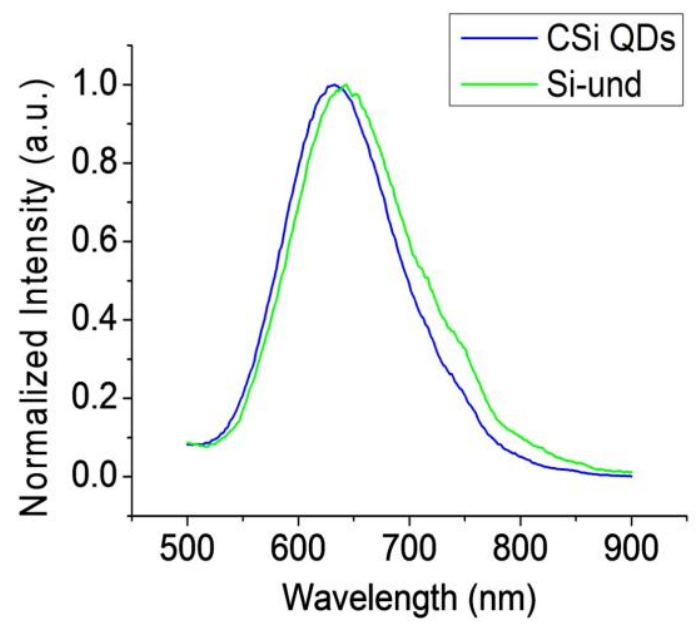

Figure 2. PL spectra of undecylenic acid functionalized SiQDs and CSiQDs 
Figure 3 shows TEM images of the undecylenic acid coated SiQDs, before and after chitosan encapsulation, along with the hydrodynamic diameter distribution of the CSiQDs from dynamic light scattering (DLS). Parts (a) and (b) of Figure 3 show TEM images of undecylenic acid functionalized SiQDs. Although high-resolution imaging is challenging because of the relatively large organic fraction in the coated particles, sub-5 nm crystalline domains are visible. Parts (c) theough (e) of Figure 3 show TEM images of the CSiQDs. The particles are spherical in shape with an average size near $70 \mathrm{~nm}$ and significant variation in size. The mean hydrodynamic diameter of chitosan-encapsulated SiQDs in water near neutral $\mathrm{pH}$ was $180 \mathrm{~nm}$, as shown in Fig. 3(f). The CSiQDs did not appear to be aggregated; their dispersion is stabilized by the positive charge of the chitosan. The size is large for some biological applications; however, it is within the size range for some promising applications such as tumor targeting. 

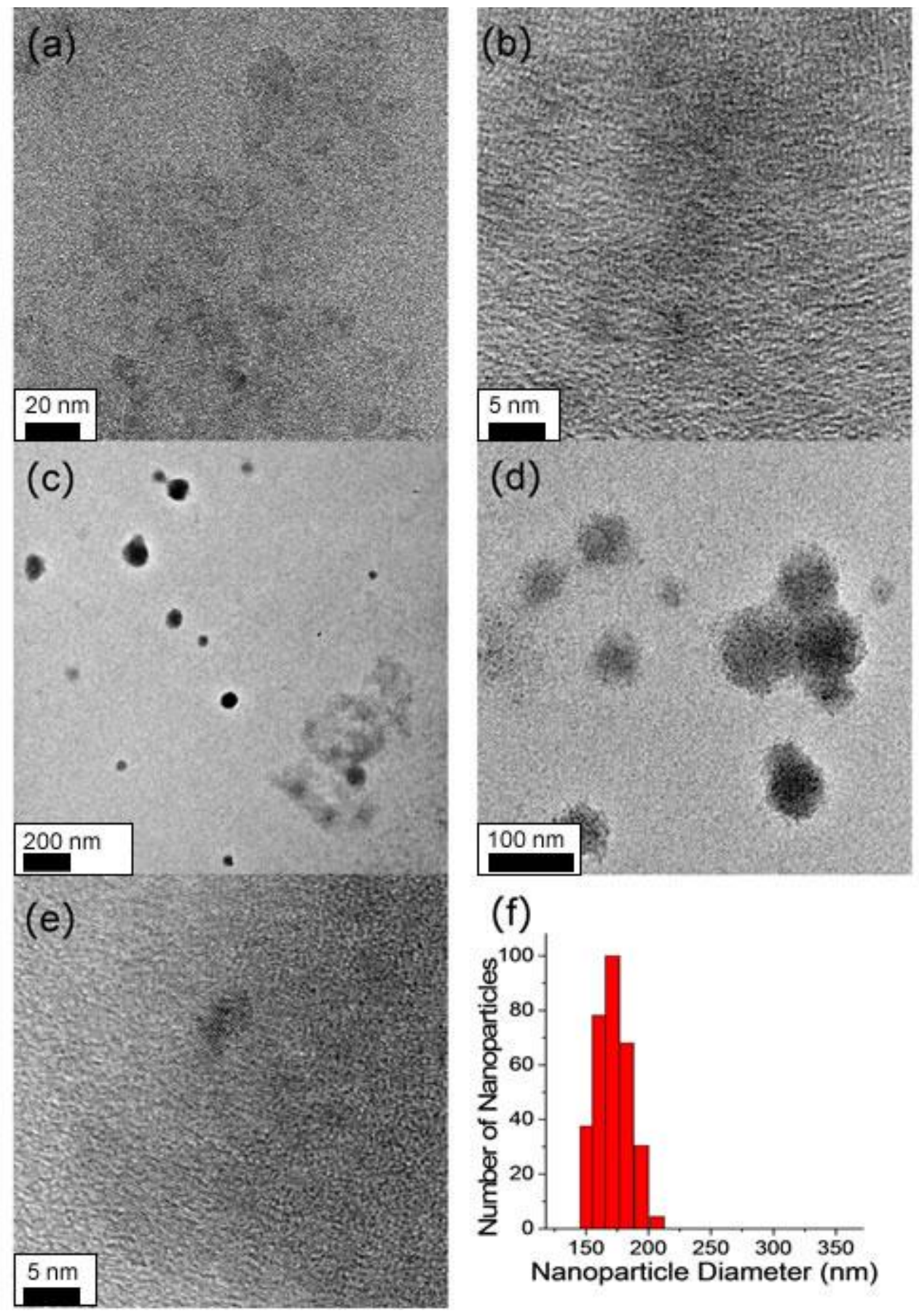

Figure 3. TEM images of (a, b) undecylenic acid terminated SiQDs, and (c, d, e) chitosan-encapsulated SiQDs. (f)The hydrodynamic diameter distribution of chitosan-encapsulated SiQDs as measured by DLS. The mean diameter is $180 \mathrm{~nm}$. 
Figure 4(a) shows the stability of the PL intensity as a function of solution $\mathrm{pH}$. The CSiQDs showed excellent stability of their photoluminescence from $\mathrm{pH} 1$ to 12 . The PL varied by less than $10 \%$ over this $\mathrm{pH}$ range. This was unexpected because chitosan itself is normally unstable at basic conditions. At a pH of 13 , however, the particles lost $98 \%$ of their luminescence. This wide pH range of PL stability may result from a synergistic relationship between chitosan and silicon nanoparticles that prevents rapid degradation. The stability of the PL from CSiQDs after heating to different temperatures is shown in Fig. 4(b). The PL decreased linearly with increasing temperature, however at the physiological temperature of $37{ }^{\circ} \mathrm{C}$ the particles maintain over $80 \%$ of their room-temperature luminescence. The particles partially regain their luminescence when cooled back to room temperature, indicating that ther may be increased non-radiative decay at hihger temperatures. Figure 4(c) confirmed the biodegradability of the CSiQDs. The results show the PL intensity of CSiQDs under three different conditions. One was held at $4{ }^{\circ} \mathrm{C}$, the other two were incubated at $37^{\circ} \mathrm{C}$ in water and PBS buffer, respectively. The refrigerated sample retained $96.2 \%$ of its PL intensity after three days, and the sample which was incubated in water maintained $86.8 \%$ of its intensity after three days. However, the PL intensity of the sample incubated in PBS buffer for three days was only $9.7 \%$ of its initial intensity. This suggests that the CSiQDs can be stored at $4{ }^{\circ} \mathrm{C}$ for a significant time while retaining their PL, but that when inserted into a biological system they will degrade within a few days. Particles (without the chitosan coating) terminated with undecylenic acid maintain their PL in ethanol for months. 
(a)

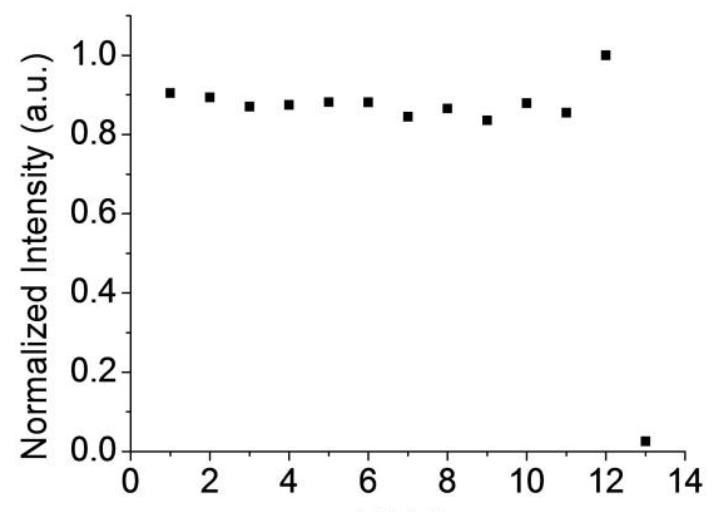

(b)

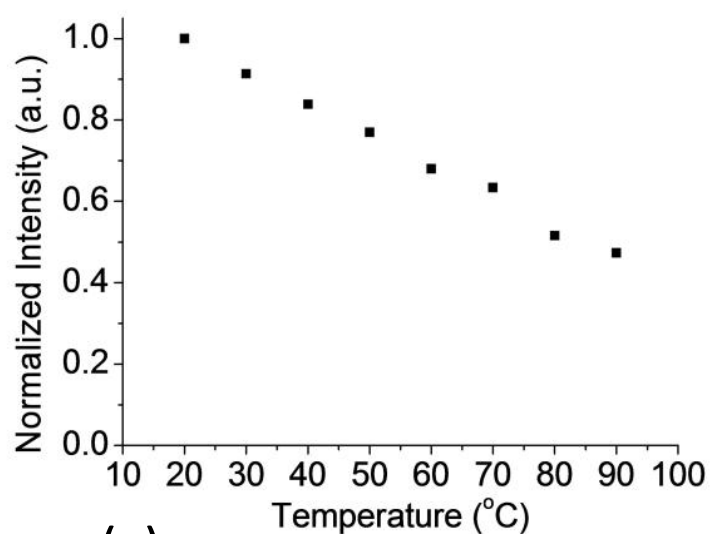

(c)

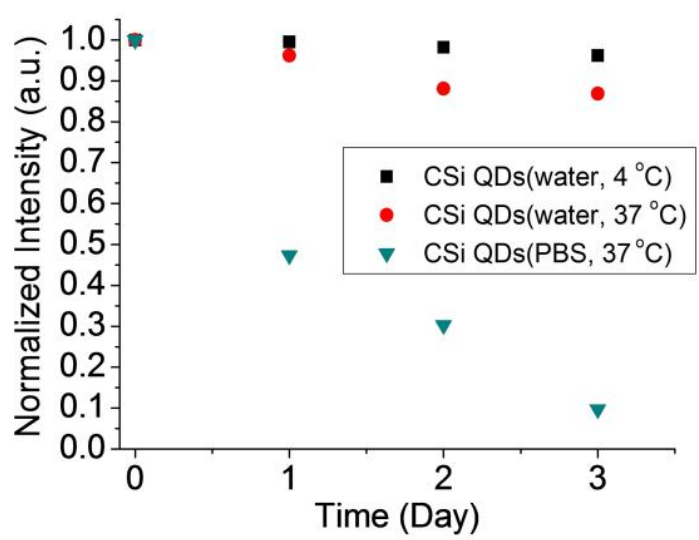

Figure 4. (a) The PL stability of CSiQDs vs. solution pH. (b) The PL stability of CSiQDs after heating to different temperatures. (c) The normalized PL intensity of CSiQDs stored under different conditions (4 ${ }^{\circ} \mathrm{C}$ in water, black squares; $37{ }^{\circ} \mathrm{C}$ in water, red circles; $37{ }^{\circ} \mathrm{C}$ in phosphorous buffered saline, cyan triangles). 
To demonstrate the utility of CSiQDs for bioimaging, they were incubated with macrophages and then imaged. Macrophages, RAW264.7, were prepared in Dulbecco minimum essential media (DMEM) with $10 \%$ fetal bovine serum (FBS), 1\% penicillin, and 1\% amphotericin $\mathrm{B}$. The day before nanoparticle treatment, cells were seeded in $35 \mathrm{~mm}$ culture dishes. On the treatment day, the cells, at a confluency of $70-80 \%$ in serum-supplemented media, were treated with the nanoparticles at a specific concentration $\left(100 \mu \mathrm{L} / \mathrm{ml}\right.$ media of $2.5 \mu \mathrm{g} / \mathrm{mL} \mathrm{CSi}$ QD dispersion) for $24 \mathrm{~h}$ at $37^{\circ} \mathrm{C} .^{25}$ For imaging, the excitation wavelength was the shortest wavelength available on the microscope $(405 \mathrm{~nm})$. Figure 5 shows that CSiQDs have stained the macrophages in these experiments.

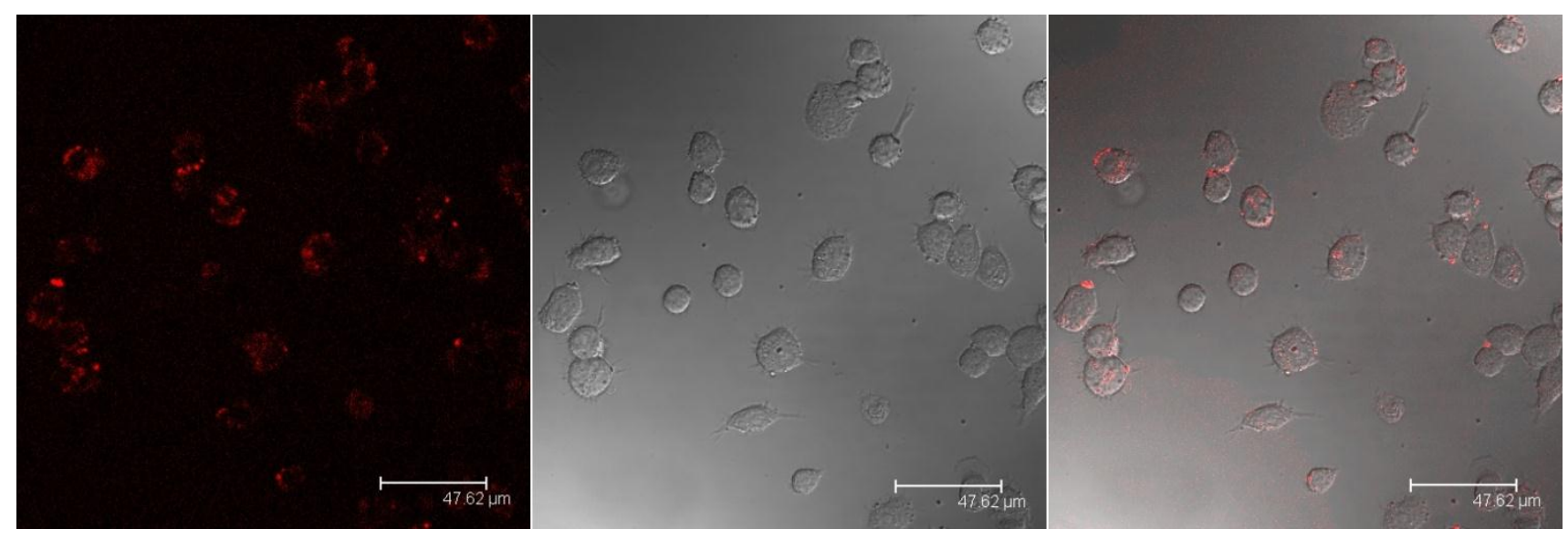

Figure 5. Laser confocal microscopy images of macrophages labeled by CSiQDs using single photon (405 nm) excitation. Fluorescence (left), transmission (center) and overlaid (right) images are shown.

We previously evaluated two-photon excitation of silicon quantum dots in chloroform and water by using femtosecond laser pulses with a wavelength of $778 \mathrm{~nm}$ and pulse duration of approximately $160 \mathrm{fs}^{26}$. There was no appreciable difference in the photoluminescence spectral distributions when comparing one- and two-photon excitation of the silicon nanoparticles. Figure 6 shows two-photon excited luminescence images of RAW macrophages labeled with CSiQDs. The absorbance of the silicon quantum dots that emit at 650 decreases drastically from the UV to the blue range ${ }^{26}$, therefore much 
brighter images can be expected by using shorter wavelengths than 900nm. The Two-photon excitation is advantageous for in vivo imaging because transmission through tissues is maximized at the near-IR wavelengths. The two-photon imaging technique can also be applied for long-term cellular imaging instead of single photon excitation, where the longer wavelengths will result in reduced photodamage compared to single-photon UV-excited imaging.

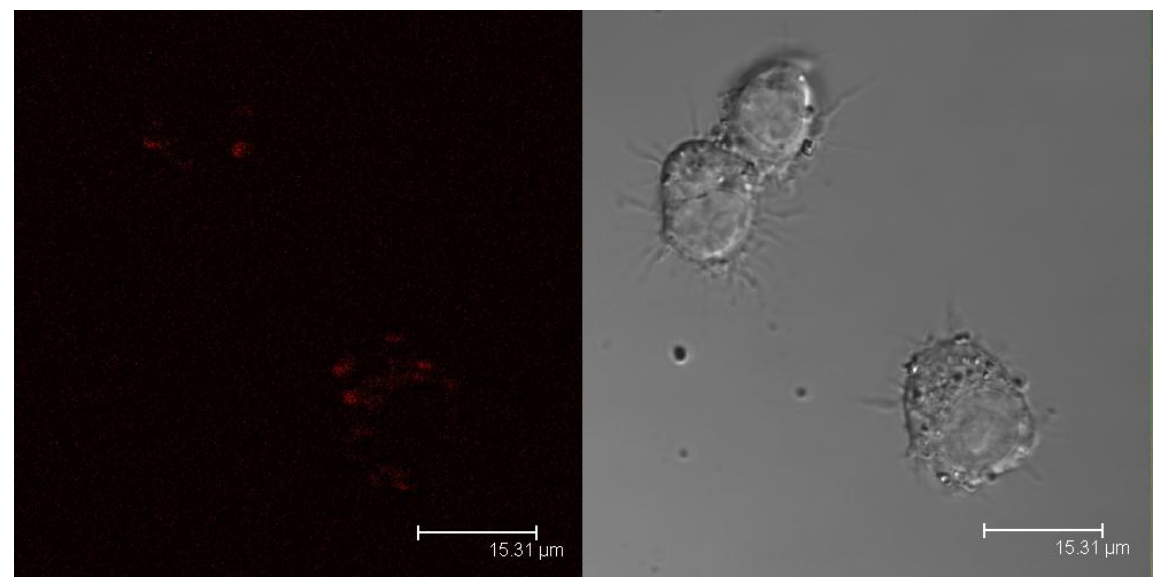

Figure 6. Laser confocal microscopy images of macrophages labeled by CSiQDs using two photon (900 $\mathrm{nm}$ ) excitation. Fluorescence (left), andtransmission (right) images are shown

\section{Conclusion.}

In conclusion, we have demonstrated a new approach for preparing CSiQDs that form stable dispersions in aqueous biological media and can be used in single-photon and two-photon excited bioimaging applications. The CSiQDs have impressive emission stability over a broad $\mathrm{pH}$ range and provided bright and clear cellular labeling on macrophages. Two-photon excitation was also demonstrated, increasing the potential for use of these CSiQDs for in vivo imaging and long-term continuous cellular imaging studies. The excess positive charges from chitosan could be used for electrostatic binding of specific targeting agents for bioapplications.

\section{Experimental Section}


Materials. Silane ( $\mathrm{SiH}_{4}$, Voltaix, electronic grade, 99.999\%), hydrofluoric acid (HF, Acros Organic, 48-51\%), nitric acid $\left(\mathrm{HNO}_{3}, \mathrm{EMD}, 68-70 \%\right)$, undecylenic acid (Acros Organic, 99\%), low molecular weight chitosan (Aldrich, 75-85\% deacetylated), and acetic acid (J. T. Baker, 100\%) were used as received. Non-luminescent Si nanoparticle powder was obtained by high temperature laser pyrolysis of silane gas in an aerosol reactor as reported previously. ${ }^{27}$ The silicon nanoparticles were collected without exposure to air and handled in a glove box to prevent surface oxidation. The primary particle diameter of the aggregated silicon powder was between 5 and $10 \mathrm{~nm}$.

Chemical Etching. To obtain photoluminescent SiQDs, a chemical etching procedure developed by Hua et $\mathrm{al}^{28}$ was adopted to obtain hydrogen terminated silicon quantum dots of the desired photoluminescence emission color. First, $300 \mathrm{mg}$ of Si nanoparticles powder was dispersed in $30 \mathrm{~mL}$ of methanol by sonication. A hydrofluoric acid (HF, $48 \mathrm{wt} \%) /$ nitric acid $\left(\mathrm{HNO}_{3}, 69 \mathrm{wt} \%\right)$ mixture (10/1, $\mathrm{v} / \mathrm{v}, 110 \mathrm{~mL}$ total) was prepared for the chemical etching process. The acid mixture was added to the $\mathrm{Si} /$ methanol dispersion and stirred for about 1-2 minutes depending on the desired luminescence emission color. To terminate the etching procedure the mixture was diluted into $400 \mathrm{~mL}$ of methanol. Luminescent SiQDs were collected using a pressure filter with poly(vinylidene fluoride) (PVDF) membrane filter and washed with $750 \mathrm{~mL}$ of $1: 3$ methanol/water mixture to remove any acid residue. Finally, SiQDs were rinsed with $50 \mathrm{~mL}$ of methanol then transferred into $20 \mathrm{~mL}$ of undecylenic acid. The etching processes were performed in a nitrogen-filled glove box to reduce SiQD oxidation and maintain hydrogen termination after etching.

Preparation of Carboxyl-Terminated SiQDs. The Si/undecylenic acid mixture was then transferred to a reflux system with addition of $20 \mathrm{~mL}$ of ethanol. Three freeze-pump-thaw cycles were used to remove dissolved oxygen from the mixture before reaction. The Si QD-undecylenic acid mixture was heated to $78.9^{\circ} \mathrm{C}$ for three hours with stirring under an inert argon environment. During the reaction, the double bond on undecylenic acid reacted with the hydrogen-terminated silicon by hydrosilylation to give a Si-C linkage. Upon surface modification, the solution changed from cloudy to clear, qualitatively confirming that the modified SiQDs could be stably dispersed in ethanol, as well as most non-polar solvents such as 
chloroform, hexane and toluene. Excess undecylenic acid was removed by dialysis of the product against $3 \mathrm{~L}$ of ethanol three times. The product after the dialysis is a dispersion of undecylenic acid functionalized SiQDs (Si-und) in ethanol.

Chitosan Encapsulation. Low molecular weight chitosan $(0.01 \% \mathrm{w} / \mathrm{v})$ was dissolved in 1.5 vol\% acetic acid to create a stock solution. The encapsulation was carried out by adding $5 \mathrm{~mL}$ of chitosan/acetic acid solution into $5 \mathrm{~mL} \mathrm{Si-und/ethanol} \mathrm{and} \mathrm{stirring} \mathrm{overnight.} \mathrm{CSiQDs} \mathrm{were} \mathrm{separated} \mathrm{by}$ centrifugation at 13.3K RPM for 1 hour, then redispersed in HPLC water and stored in the refrigerator at $\sim 4{ }^{\circ} \mathrm{C}$. Dialysis against water could be used to remove remaining traces of ethanol or acetic acid.

Electron Microscopy. The silicon nanoparticles were characterized by transmission electron microscopy (TEM, JEOL 2010) before and after encapsulation. Samples were drop-cast from ethanolic or aqueous dispersions onto a TEM grid and the water was allowed to evaporate at room temperature.

Photoluminescence Spectra. Photoluminescence spectra were measured with a Perkin-Elmer LS 50 fluorescence spectrometer with a $351 \mathrm{~nm}$ band-pass filter used to suppress any scattered light from the source. The excitation wavelength was $350 \mathrm{~nm}$. The emission cutoff was set to $390 \mathrm{~nm}$ for most of the PL measurements shown here. The $\mathrm{pH}$ of Si QD dispersions was varied by addition of $\mathrm{NaOH}$ or $\mathrm{HCl}$.

Dynamic Light Scattering. The hydrodynamic diameter and zeta potential were measured by dynamic light scattering (DLS, BIC, 90 Plus).

Cellular Imaging. The uptake of CSiQDs into macrophages was confirmed by confocal imaging (Leica Microsystems Semiconductor GmbH, Wetzlerm Germany, with single photon laser excitation at $405 \mathrm{~nm}$ or two-photon laser excitation at $900 \mathrm{~nm}$ ).

Acknowledgements. This study was supported by grants from the NCI RO1CA119397, and the John R. Oishei Foundation.

\section{References Cited.}


1. Prasad, P. N., Biophotonics. Wiley-Interscience: Hoboken, NJ, 2003.

2. $\quad$ Prasad, P. N., Nanophotonics. Wiley-Interscience: Hoboken, NJ, 2004.

3. Rzigalinski, B. A.; Strobl, J. S., Toxicology and Applied Pharmacology 2009, 238, 280-288.

4. Hauck, T. S.; Anderson, R. E.; Fischer, H. C.; Newbigging, S.; Chan, W. C. W., Small 2010, 6, 138-144.

5. $\quad$ Kim, S.; Lim, Y. T.; Soltesz, E. G.; De Grand, A. M.; Lee, J.; Nakayama, A.; Parker, J. A.; Mihaljevic, T.; Laurence, R. G.; Dor, D. M.; Cohn, L. H.; Bawendi, M. G.; Frangioni, J. V., Nat Biotech 2004, 22, 93-97.

6. $\quad$ Gao, X.; Cui, Y.; Levenson, R. M.; Chung, L. W. K.; Nie, S., Nat Biotech 2004, 22, 969-976.

7. Soo Choi, H.; Liu, W.; Misra, P.; Tanaka, E.; Zimmer, J. P.; Itty Ipe, B.; Bawendi, M. G.; Frangioni, J. V., Nat Biotech 2007, 25, 1165-1170.

8. $\quad$ Choi, J.; Wang, N. S.; Reipa, V., Bioconjugate Chemistry 2008, 19, 680-685.

9. $\quad$ Erogbogbo, F.; Yong, K.-T.; Roy, I.; Xu, G.; Prasad, P. N.; Swihart, M. T., ACS Nano 2008, 2, 873-878.

10. Mangolini, L.; Jurbergs, D.; Rogojina, E.; Kortshagen, U., physica status solidi (c) 2006, 3, 3975-3978.

11. Tu, C.; Ma, X.; Pantazis, P.; Kauzlarich, S. M.; Louie, A. Y., Journal of the American Chemical Society 2010, 132, 2016-2023.

12. Warner, J. H.; Hoshino, A.; Yamamoto, K.; Tilley, R. D., Angewandte Chemie International Edition 2005, 44, 4550-4554.

13. Park, J.-H.; Gu, L.; von Maltzahn, G.; Ruoslahti, E.; Bhatia, S. N.; Sailor, M. J., Nat Mater 2009, 8, 331-336.

14. Fujioka, K.; Hiruoka, M.; Sato, K.; Manabe, N.; Miyasaka, R.; Hanada, S.; Hoshino, A.; Tilley, R. D.; Manome, Y.; Hirakuri, K.; Yamamoto, K., Nanotechnology 2008, 19, 415102.

15. Erogbogbo, F.; Yong, K.-T.; Roy, I.; Hu, R.; Law, W.-C.; Zhao, W.; Ding, H.; Wu, F.; Kumar, R.; Swihart, M. T.; Prasad, P. N., ACS Nano 2010, ASAP article.

16. Kumar, M. N. V. R.; Muzzarelli, R. A. A.; Muzzarelli, C.; Sashiwa, H.; Domb, A. J., Chemical Reviews 2004, 104, 6017-6084.

17. Lavertu, M.; Méthot, S.; Tran-Khanh, N.; Buschmann, M. D., Biomaterials 2006, 27, 48154824.

18. Mansouri, S.; Lavigne, P.; Corsi, K.; Benderdour, M.; Beaumont, E.; Fernandes, J. C., European Journal of Pharmaceutics and Biopharmaceutics 2004, 57, 1-8.

19. Vila, A.; Sánchez, A.; Tobío, M.; Calvo, P.; Alonso, M. J., Journal of Controlled Release 2002, $78,15-24$.

20. Mitra, S.; Gaur, U.; Ghosh, P. C.; Maitra, A. N., Journal of Controlled Release 2001, 74, 317323.

21. Tan, W. B.; Zhang, Y., Advanced Materials 2005, 17, 2375-2380.

22. Xie, M.; Liu, H. H.; Chen, P.; Zhang, Z. L.; Wang, X. H.; Xie, Z. X.; Du, Y. M.; Pan, B. Q.; Pang, D. W., Chem. Commun. 2005, 5518-5520.

23. Clark, R. J.; Dang, M. K. M.; Veinot, J. G. C., Langmuir 2010, 26, 15657-15664.

24. Erogbogbo, F.; Tien, C.-A.; Chang, C.-W.; Yong, K.-T.; Ding, H.; Law, W.-C.; Roy, I.; Swihart, M. T.; Prasad, P. N., Bioconjugate Chemistry 2011, Accepted.

25. Kumar, R.; Ding, H.; Hu, R.; Yong, K.-T.; Roy, I.; Bergey, E. J.; Prasad, P. N., Chemistry of Materials 2010, 22, 2261-2267.

26. He, G. S.; Zheng, Q.; Yong, K.-T.; Erogbogbo, F.; Swihart, M. T.; Prasad, P. N., Nano Letters 2008, 8, 2688-2692.

27. Li, X.; He, Y.; Talukdar, S. S.; Swihart, M. T., Langmuir 2003, 19, 8490-8496.

28. Hua, F.; Swihart, M. T.; Ruckenstein, E., Langmuir 2005, 21, 6054-6062. 
Table of Content Image:

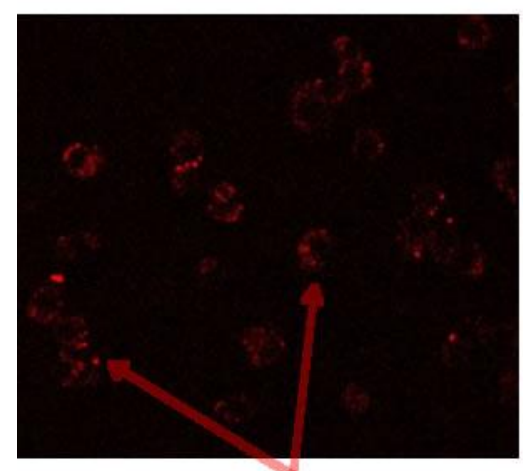

Luminescent Si QDS in Cells

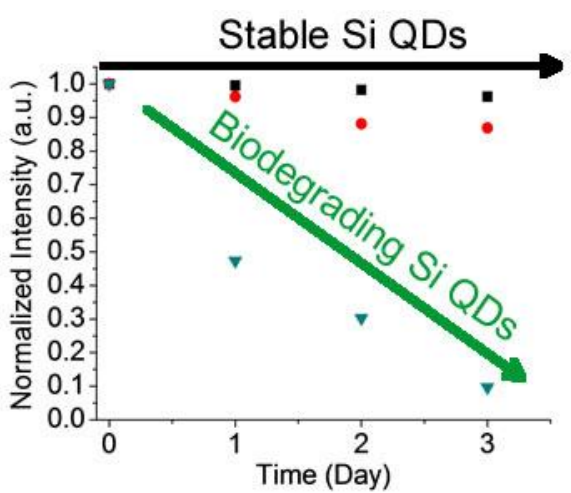

Biodegradation over time 